\title{
Elementary vortex pinning potential in superconductors with unconventional order parameter
}

\author{
Nobuhiko Hayashi ${ }^{\mathrm{a}, *}$ and Yusuke Kato ${ }^{\mathrm{b}}$ \\ ${ }^{a}$ Computer Center, Okayama University, Okayama 700-8530, Japan \\ b Department of Basic Science, University of Tokyo, Tokyo 153-8902, Japan
}

\begin{abstract}
The elementary vortex pinning potential is studied in unconventional superconductors within the framework of the quasiclassical theory of superconductivity. Numerical results are presented for $d$-, anisotropic $s$-, and isotropic $s$-wave superconductors to show explicitly that in unconventional superconductors the vortex pinning potential is determined mainly by the loss of the condensation energy in bulk due to the presence of the pinning center, i.e., by the breakdown of the Anderson's theorem. It is found that the vortex pinning energy in the $d$-wave pairing case is $4-13$ times larger than those in the $s$-wave pairing cases. This means that an enhancement of pinning effect in unconventional superconductors occurs due to the breakdown of the Anderson's theorem. The case of a chiral $p$-wave superconductor is also investigated in terms of the vortex core states subject to the Andreev reflection, where important is whether the vorticity and chirality are parallel or antiparallel.
\end{abstract}

Key words: vortex pinning, unconventional superconductor, $d$-wave pairing, chiral $p$-wave pairing

PACS: $74.60 . \mathrm{Ge}$

\section{Introduction}

Much attention has been focused on the vortex pinning in type-II superconductors. The vortex pinning in the superconductors under magnetic fields plays an important role on various vortex-related quantities and phenomena such as the critical current, hysteresis of the magnetization, and quantum vortex

\footnotetext{
* Corresponding author. Fax: +81-86-251-7244

Email address: hayashi@mp.okayama-u.ac.jp (Nobuhiko Hayashi).
} 
tunneling. The problem of the vortex pinning is categorized in two aspects. One is the elementary vortex pinning force, which is the interaction between a vortex and a single defect. The other is the summation problem, which is how the elementary vortex pinning forces add up to a holding force on the elastic vortex lattice under a distribution of defects [1]. A study of the elementary vortex pinning is the step necessary for the understanding of the phenomena related to the vortex pinning in the superconductors under magnetic fields.

Traditionally, the mechanism of the elementary vortex pinning is considered to be that a defect locally prohibits superconducting condensation so that it attracts a normal region of a vortex core in order to avoid the loss of the condensation energy. Thuneberg et al. [2,3] advanced the understanding of the mechanism of the elementary vortex pinning, taking account of a nonlocal effect that a defect as a scattering center scatters the quasiparticles. This mechanism of the vortex pinning is that the quasiparticle scattering by the defect inside a vortex core helps to avoid suppressions of the order parameter around the vortex center up to distances of the order of the coherence length.

In this paper, we investigate the elementary vortex pinning potential in unconventional superconductors, following the analysis started by Thuneberg et al. [2,3] for conventional $s$-wave superconductors. The unconventional superconductivity is recently proposed for many superconductors such as high- $T_{\mathrm{c}}$ cuprates, organic conductors, and heavy-fermion compounds. For example, the $d$-wave superconductivity is believed to be realized in most of the high$T_{\mathrm{c}}$ cuprates, and the chiral $p$-wave one is expected in a non-copper-layered perovskite superconductor $\mathrm{Sr}_{2} \mathrm{RuO}_{4}$ [4] (although it seems to be open to further discussion [5]). We calculate numerically the elementary vortex pinning potential for $d$-, anisotropic $s^{-}$, and isotropic $s$-wave pairings, and compare them each other to clarify what could be one of the characteristic properties specific to the superconductors with the unconventional order parameter. We also present an analytical consideration of a novel chirality-dependent vortex pinning in a chiral $p$-wave superconductor.

\section{Formulation}

To investigate the vortex pinning, we use the quasiclassical theory of superconductivity [6]. We start with the Eilenberger equation for the quasiclassical Green function in the absence of the pinning,

$$
\hat{g}_{\mathrm{imt}}\left(i \omega_{n}, \mathbf{r}, \overline{\mathbf{k}}\right)=-i \pi\left(\begin{array}{cc}
g_{\mathrm{imt}} & i f_{\mathrm{imt}} \\
-i f_{\mathrm{imt}}^{\dagger} & -g_{\mathrm{imt}}
\end{array}\right)
$$

namely, 


$$
i v_{\mathrm{F}} \overline{\mathbf{k}} \cdot \nabla \hat{g}_{\mathrm{imt}}+\left[i \omega_{n} \hat{\tau}_{3}-\hat{\Delta}, \hat{g}_{\mathrm{imt}}\right]=0,
$$

where $\hat{\tau}_{3}$ is the Pauli matrix and the order parameter

$$
\hat{\Delta}(\mathbf{r}, \overline{\mathbf{k}})=\left(\begin{array}{cc}
0 & \Delta(\mathbf{r}, \overline{\mathbf{k}}) \\
-\Delta^{*}(\mathbf{r}, \overline{\mathbf{k}}) & 0
\end{array}\right) .
$$

The Eilenberger equation (2) is supplemented by the normalization condition $\hat{g}_{\text {imt }}\left(i \omega_{n}, \mathbf{r}, \overline{\mathbf{k}}\right)^{2}=-\pi^{2} \hat{1}$, and the commutator is $[\hat{a}, \hat{b}]=\hat{a} \hat{b}-\hat{b} \hat{a}$. The symbol $\mathbf{r}=(r \cos \phi, r \sin \phi)$ denotes the center of mass coordinate of the Cooper pairs and $\overline{\mathbf{k}}=(\cos \theta, \sin \theta)$ denotes the relative coordinate of them. The cylindrical Fermi surface is assumed. We use units in which $\hbar=k_{\mathrm{B}}=1$.

Following Thuneberg et al. $[2,3,7]$, the effect of the pinning is introduced as follows to the quasiclassical theory of superconductivity. The quasiclassical Green function $\hat{g}$ in the presence of a point-like non-magnetic defect situated at $\mathbf{r}=\mathbf{R}$ is obtained from the Eilenberger equation

$$
i v_{\mathrm{F}} \overline{\mathbf{k}} \cdot \nabla \hat{g}+\left[i \omega_{n} \hat{\tau}_{3}-\hat{\Delta}, \hat{g}\right]=\left[\hat{t}, \hat{g}_{\mathrm{imt}}\right] \delta\left(\mathbf{r}^{\prime}\right),
$$

and the $t$ matrix due to the defect

$$
\hat{t}\left(i \omega_{n}, \mathbf{r}^{\prime}\right)=\frac{v}{D}\left[\hat{1}+N_{0} v\left\langle\hat{g}_{\mathrm{imt}}\left(i \omega_{n}, \mathbf{r}^{\prime}, \overline{\mathbf{k}}\right)\right\rangle_{\theta}\right],
$$

where $\mathbf{r}^{\prime}=\mathbf{r}-\mathbf{R}$, the denominator $D=1+\left(\pi N_{0} v\right)^{2}\left[\left\langle g_{\text {imt }}\right\rangle_{\theta}^{2}+\left\langle f_{\text {imt }}\right\rangle_{\theta}\left\langle f_{\text {imt }}^{\dagger}\right\rangle_{\theta}\right]$, the symbol $\langle\cdots\rangle_{\theta}=\int \cdots d \theta / 2 \pi$, the normal-state density of states on the Fermi surface is $N_{0}$, and we assume the $s$-wave scattering $v$ to obtain Eq. (5). The cross section of the defect is given by $\sigma_{\mathrm{tr}}=\left(4 \pi / k_{\mathrm{F}}^{2}\right) \sin ^{2} \delta_{0}$ with the scattering phase shift $\delta_{0}$, and $\tan \delta_{0}=-\pi N_{0} v$.

The free energy in the presence of the defect is, at the temperature $T$, given as $[2,3,7]$

$$
\delta \Omega(\mathbf{R})=N_{0} T \int_{0}^{1} d \lambda \sum_{\omega_{n}} \int d \overline{\mathbf{k}} \int d \mathbf{r} \operatorname{Tr}\left[\delta \hat{g}_{\lambda} \hat{\Delta}_{b}\right]
$$

where $\delta \hat{g}_{\lambda}=\hat{g}-\hat{g}_{\text {imt }}$ is evaluated at $\hat{\Delta}=\lambda \hat{\Delta}_{b}$, and $\hat{\Delta}_{b}$ is the order parameter in the absence of the defect. Equation (6) represents the difference in the free energy between the states with and without the defect, and then gives the vortex pinning potential $\delta \Omega(\mathbf{R})$. 

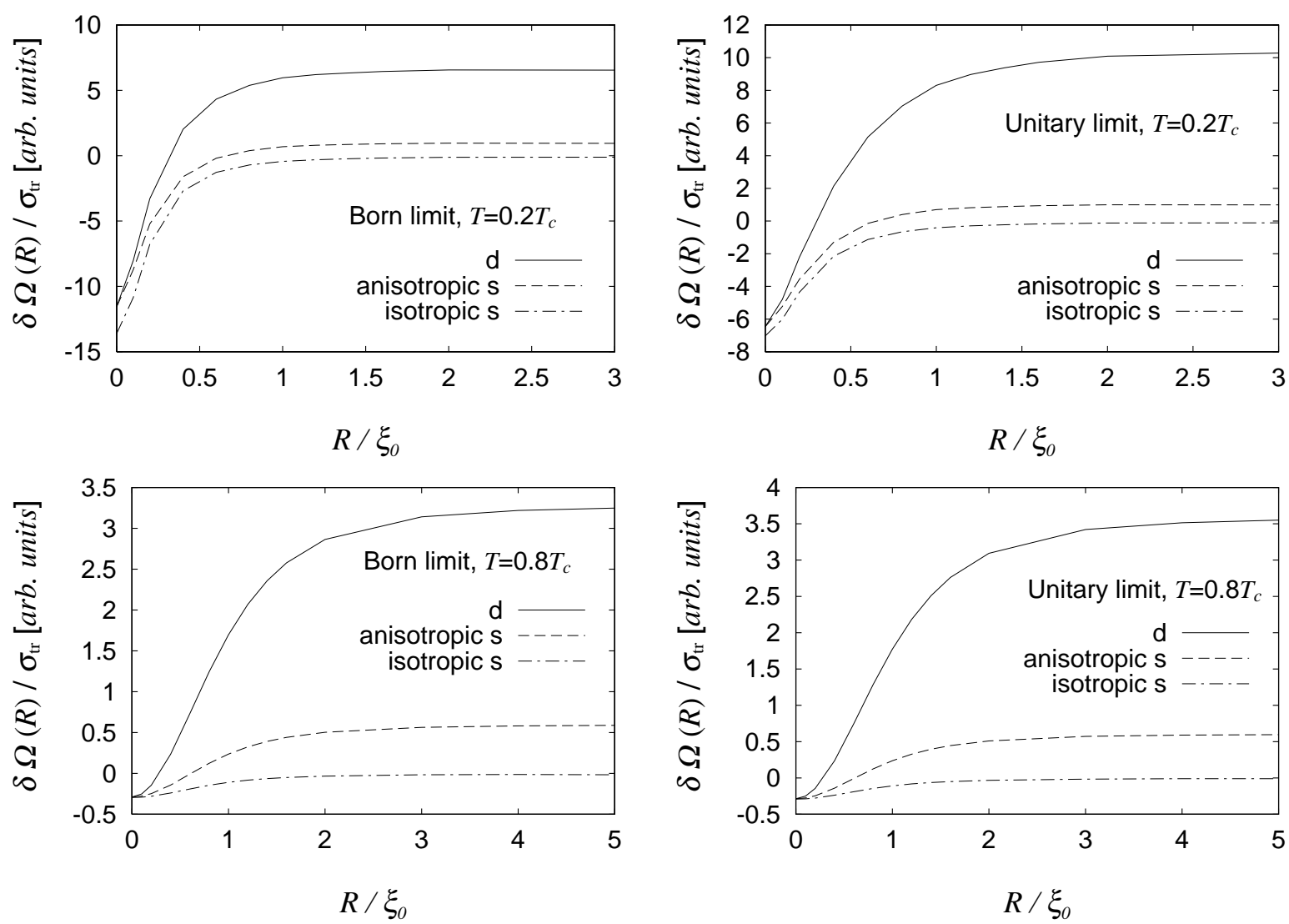

Fig. 1. The vortex pinning potential as a function of the distance $R$ between the vortex center and the defect. The defect is situated on the $x$ axis (in the direction of $\phi=0)$.

\section{Results}

\section{$3.1 d$-, anisotropic s-, and isotropic s-wave pairings}

We calculate the vortex pinning potential $\delta \Omega(\mathbf{R})$ numerically with the order parameter around a vortex, $\Delta_{b}(\mathbf{r}, \overline{\mathbf{k}})=F(\theta) \Delta_{\mathrm{BCS}}(T) \tanh [r / \xi(T)] \exp (i \phi)$, (note that the vortex center is situated at $\mathbf{r}=0$ ). Here, the $\mathbf{k}$-dependent function $F(\theta)=1$ for isotropic $s$-wave, $\sqrt{2}|\cos 2 \theta|$ for anisotropic $s$-wave, and $\sqrt{2} \cos 2 \theta$ for $d$-wave pairings. $\Delta_{\mathrm{BCS}}(T)$ is the BCS order parameter amplitude and $\xi(T)$ the coherence length. $\left(\xi(T)=\xi_{0} \cdot\left[\Delta_{0} / \Delta_{\mathrm{BCS}}(T)\right], \Delta_{0}=\Delta_{\mathrm{BCS}}(0)\right.$, and $\left.\xi_{0}=v_{\mathrm{F}} / \Delta_{0}.\right)$

In Fig. 1, we show the numerical results for the Born limit $\left(\delta_{0} \rightarrow 0\right)$ and the unitary limit $\left(\delta_{0}=\pi / 2\right)$. The defect is situated on the $x$ axis (in the direction of $\phi=0)$. In the case of the isotropic $s$-wave pairing, when the defect is situated in bulk, i.e., far away from the vortex center $(R=|\mathbf{R}| \rightarrow \infty)$, the difference in

the free energy between the states with and without the defect, $\delta \Omega(R)$, is equal 
to zero. This is because the Anderson's theorem [8] is valid for homogeneous systems in isotropic $s$-wave superconductors and the non-magnetic defect has no influence on the free energy. On the other hand, in the anisotropic pairing cases, $\delta \Omega(R \rightarrow \infty)$ is finite and positive as seen in Fig. 1, which is the loss of the condensation energy in bulk due to the presence of the defect and to the breakdown of the Anderson's theorem. The condensation energy loss in bulk $\delta \Omega(R \rightarrow \infty)$ contributes to the depth of the vortex pinning potential $\delta \Omega(R)$, i.e., the vortex pinning energy. While the contribution of $\delta \Omega(R \rightarrow \infty)$ to the vortex pinning energy was first pointed out by Thuneberg et al. [7] in the context of an ion in $p$-wave superfluid ${ }^{3} \mathrm{He}-\mathrm{B}$, we performed here a numerical calculation explicitly for the vortex pinning potential in the $d$-wave superconductors. It is noted from Figs. 1(c) and 1(d) that, at $T=0.8 T_{\mathrm{c}}$ (high temperature), the vortex pinning energy in the $d$-wave case is dominantly determined by the condensation energy loss in bulk $\delta \Omega(R \rightarrow \infty)$, and is $4-$ 13 times larger than those in the $s$-wave cases.

\section{2 chiral p-wave pairing}

For the chiral $p$-wave pairing $\mathbf{d}=\mathbf{z}\left(\bar{k}_{x} \pm i \bar{k}_{y}\right)$ [4], we analytically investigate the vortex pinning potential. In the case of that chiral $p$-wave pairing, there exist the following two possible forms for the order parameter $\Delta(r, \phi ; \theta)(=$ $\Delta(\mathbf{r}, \overline{\mathbf{k}}))$ of axisymmetric vortex [9-11]: (i) $\Delta_{b}^{+-}(r, \phi ; \theta)=\Delta_{+}(r) \exp [i(\theta-$ $\phi)]+\Delta_{-}(r) \exp [i(-\theta+\phi)]$, where the chirality and vorticity are antiparallel; (ii) $\Delta_{b}^{++}(r, \phi ; \theta)=\Delta_{+}(r) \exp [i(\theta+\phi)]+\Delta_{-}(r) \exp [i(-\theta+3 \phi)]$, where the chirality and vorticity are parallel. Here, $\Delta_{ \pm}(r)$ are real, $\Delta_{+}(r \rightarrow \infty)=\Delta_{\mathrm{BCS}}(T)$, and $\Delta_{-}(r \rightarrow \infty)=0$.

We focus on $\delta \Omega(R=0)$, where both the defect and the vortex center are situated just at the origin $\mathbf{r}=0$. The quasiparticles inside the vortex core, subject to the Andreev reflection, run along straight lines, namely the quasiclassical trajectories $[11,12]$. We consider the quasiclassical trajectories which go through the origin $\mathbf{r}=0$. On those trajectories with zero impact parameter, the position vector is parallel to the direction of the trajectory (i.e., $\mathbf{r} \| \overline{\mathbf{k}}$ ), and therefore $\phi=\theta, \theta+\pi$. In this situation the order parameter is $\Delta_{b}^{+-}(r, \phi ; \theta)= \pm\left(\Delta_{+}(r)+\Delta_{-}(r)\right)$ in the case of $(\mathrm{i})$, and $\Delta_{b}^{++}(r, \phi ; \theta)=$ $\pm\left(\Delta_{+}(r)+\Delta_{-}(r)\right) \exp (2 i \theta)$ in the case of (ii). Of importance is the difference in the phase factor of these order parameters of (i) and (ii).

On the basis of an analysis of the so-called zero-core vortex model in Ref. [3], the matrix elements of $\hat{g}_{\text {imt }}$ at the vortex center are approximately obtained as $g_{\mathrm{imt}}=\sqrt{\omega_{n}^{2}+|\tilde{\Delta}|^{2}} / \omega_{n}, f_{\mathrm{imt}}=-\tilde{\Delta} / \omega_{n}$, and $f_{\mathrm{imt}}^{\dagger}=\tilde{\Delta}^{*} / \omega_{n}$, where $\tilde{\Delta}=$ $\Delta_{b}^{+ \pm}(r \rightarrow \infty, \phi=\theta ; \theta)$. Because of the difference in the phase factor of the order parameters mentioned above, $\left\langle f_{\mathrm{imt}}\right\rangle_{\theta}=f_{\mathrm{imt}}$ and $\left\langle f_{\mathrm{imt}}^{\dagger}\right\rangle_{\theta}=f_{\mathrm{imt}}^{\dagger}$ in the 
case of (i), while $\left\langle f_{\text {imt }}\right\rangle_{\theta}=0$ and $\left\langle f_{\text {imt }}^{\dagger}\right\rangle_{\theta}=0$ in the case of (ii). Therefore, in the case of $(\mathrm{i}),\left\langle\hat{g}_{\mathrm{imt}}\right\rangle_{\theta}=\hat{g}_{\mathrm{imt}}$, and then $\left[\hat{t}, \hat{g}_{\mathrm{imt}}\right]=0$ from Eq. (5). In the case of (ii), $\left\langle\hat{g}_{\text {imt }}\right\rangle_{\theta} \neq \hat{g}_{\text {imt }}$, and then $\left[\hat{t}, \hat{g}_{\text {imt }}\right] \neq 0$.

When $\left[\hat{t}, \hat{g}_{\text {imt }}\right]=0$, the Eilenberger equation (4) in the presence of the defect is identical to Eq. (2) (the equation in the absence of the defect), i.e., the defect has no influence on the Green function and the free energy. From it and the above analysis, $\delta \Omega(0)=0$ in the case of (i) when the chirality is antiparallel to the vorticity, and $\delta \Omega(0) \neq 0$ in the case of (ii) when the sense of the chirality is the same as that of the vorticity. It means that the vortex pinning depends on the chirality in the chiral $p$-wave superconductor. This analytical result is certainly confirmed by a numerical calculation [13] with self-consistently obtained order parameters $\Delta_{b}^{+ \pm}(r, \phi ; \theta)[11]$.

\section{Summary}

We investigated the elementary vortex pinning potential $\delta \Omega(R)$ on the basis of the quasiclassical theory of superconductivity. The numerical results were presented in Fig. 1 for the $d$-wave and $s$-wave pairings. The vortex pinning energy in the $d$-wave pairing case is about 10 times larger than those in the $s$-wave pairing cases, at a high temperature, because of the breakdown of the Anderson's theorem in unconventional superconductors. We also investigated analytically the quasiparticles inside the vortex core in the chiral $p$-wave superconductor, and found the chirality-dependent vortex pinning. We expect that the chirality-dependent vortex pinning revealed in this paper shares the same physics with impurity effects on the quasiparticle relaxation time [12] and on the energy spectrum $[14,15]$ inside the vortex core in chiral $p$-wave superconductors.

\section{Acknowledgements}

One of the authors (N.H.) thanks M. Ichioka, K. Machida, M. Takigawa, N. Nakai, and M. Matsumoto for useful discussions. Y.K. thanks M. Sigrist for useful discussions. This work is partly supported by Grant-in-Aid for Scientific Research on Priority Areas (A) of "Novel Quantum Phenomena in Transition Metal Oxides" (12046225) from the Ministry of Education, Science, Sports and Culture and Grant-in-Aid for Encouragement of Young Scientists from Japan Society for the Promotion of Science (12740203). 


\section{References}

[1] H. R. Kerchner, D. K. Christen, C. E. Klabunde, S. T. Sekula, and R. R. Coltman, Jr., Phys. Rev. B 27 (1983) 5467.

[2] E. V. Thuneberg, J. Kurkijärvi, and D. Rainer, Phys. Rev. Lett. 48 (1982) 1853.

[3] E. V. Thuneberg, J. Kurkijärvi, and D. Rainer, Phys. Rev. B 29 (1984) 3913.

[4] M. Sigrist, D. Agterberg, A. Furusaki, C. Honerkamp, K. K. Ng, T. M. Rice, and M. E. Zhitomirsky, Physica C 317-318 (1999) 134.

[5] A. G. Lebed and N. Hayashi, Physica C 341-348 (2000) 1677.

[6] J. W. Serene and D. Rainer, Phys. Rep. 101 (1983) 221.

[7] E. V. Thuneberg, J. Kurkijärvi, and D. Rainer, J. Phys. C: Solid State Phys., 14 (1981) 5615; See also, M. Friesen and P. Muzikar, Phys. Rev. B 53 (1996) R11953.

[8] P. W. Anderson, J. Phys. Chem. Solids 11 (1959) 26.

[9] R. Heeb and D. F. Agterberg, Phys. Rev. B 59 (1999) 7076.

[10] M. Matsumoto and R. Heeb, cond-mat/0101155.

[11] Y. Kato and N. Hayashi, submitted to J. Phys. Soc. Jpn.; cond-mat/0107517.

[12] Y. Kato, J. Phys. Soc. Jpn. 69 (2000) 3378.

[13] N. Hayashi and Y. Kato, in preparation.

[14] G. E. Volovik, cond-mat/9709159.

[15] M. Matsumoto and M. Sigrist, Physica B 281-282 (2000) 973. 